PROCEEDINGS OF THE

AMERICAN MATHEMATICAL SOCIETY

Volume 128, Number 11, Pages 3349-3360

S 0002-9939(00)05584-2

Article electronically published on May 18, 2000

\title{
SOLVABILITY OF A FINITE OR INFINITE SYSTEM OF DISCONTINUOUS QUASIMONOTONE DIFFERENTIAL EQUATIONS
}

\author{
DANIEL C. BILES AND ERIC SCHECHTER \\ (Communicated by Hal L. Smith)
}

\begin{abstract}
This paper proves the existence of solutions to the initial value problem

(IVP) $\quad\left\{\begin{array}{l}x^{\prime}(t)=f(t, x(t)) \\ x(0)=0,\end{array} \quad(0 \leq t \leq 1)\right.$,

where $f:[0,1] \times \mathbb{R}^{M} \rightarrow \mathbb{R}^{M}$ may be discontinuous but is assumed to satisfy conditions of superposition-measurability, quasimonotonicity, quasisemicontinuity, and integrability. The set $M$ can be arbitrarily large (finite or infinite); our theorem is new even for $\operatorname{card}(M)=2$. The proof is based partly on measure-theoretic techniques used in one dimension under slightly stronger hypotheses by Rzymowski and Walachowski. Further generalizations are mentioned at the end of the paper.
\end{abstract}

\section{CONTENTS}

Our main results are presented in $\S 1$. A review of the literature is given in $\S 2$ and $\S 3$, focusing respectively on our hypotheses and conclusion. The main theorem is proved in $\S 4$. Some brief remarks on further generalizations are given in $\S 5$.

We are grateful to the referee for many suggestions which we have incorporated into this revised version.

\section{MAin RESUlts}

All integrals in this paper are understood to be Lebesgue integrals. All measurability is understood to be Lebesgue measurability - i.e., the inverse image of each Borel set is a Lebesgue-measurable set.

Let $M$ be a nonempty set (not necessarily finite or countable). Members of $\mathbb{R}^{M}$ will be written as $M$-tuples $\left(x_{\mu}, x_{\nu}, \ldots\right)$, rather than as functions $x(\mu)$; this is consistent with the usual vector notation $x=\left(x_{1}, x_{2}, \ldots, x_{n}\right)$ when $M$ is a finite set. The space $\mathbb{R}^{M}$ will be equipped with the product topology; thus a function $x(t)$ taking values in $\mathbb{R}^{M}$ is continuous if and only if each of its components $x_{\mu}(t)$ is continuous. We emphasize that when $M$ is finite, then $\mathbb{R}^{M}$ is just ordinary Euclidean space.

Received by the editors January 13, 1999.

2000 Mathematics Subject Classification. Primary 34A12, 34A40; Secondary 45G15.

Key words and phrases. Quasimonotone, semicontinuous, quasisemicontinuous, supmeasurable, subsolution. 
By a solution of (IVP) we shall mean a continuous function $x:[0,1] \rightarrow \mathbb{R}^{M}$ that satisfies $x(0)=0$, and such that each component function $x_{\mu}:[0,1] \rightarrow \mathbb{R}$ is absolutely continuous and satisfies $x_{\mu}^{\prime}(t)=f_{\mu}(t, x(t))$ almost everywhere in $[0,1]$.

Theorem 1.1. Assume that $f:[0,1] \times \mathbb{R}^{M} \rightarrow \mathbb{R}^{M}$ satisfies these conditions:

(i) $f$ is superposition-measurable, in this sense: Whenever $x:[0,1] \rightarrow \mathbb{R}^{M}$ is a continuous function, then each of the real-valued functions $t \mapsto f_{\mu}(t, x(t))$ (for $\mu \in M$ ) is measurable.

(ii) For each $t \in[0,1]$, the function $f(t, \cdot): \mathbb{R}^{M} \rightarrow \mathbb{R}^{M}$ is quasisemicontinuous that is, for each $\mu \in M$ and $x \in \mathbb{R}^{M}$,

$$
\limsup _{y \uparrow x} f_{\mu}(t, y) \leq f_{\mu}(t, x) \leq \liminf _{y \downarrow x} f_{\mu}(t, y) .
$$

Here $\lim \sup _{y \uparrow x} f_{\mu}(t, y)$ means the supremum of all the limits of convergent sequences of the form $f_{\mu}\left(t, y_{n}\right)$, where $\left(y_{n}\right)$ is a sequence in $\mathbb{R}^{M}$ that increases to $x$ in every component. We define $\liminf _{y \downarrow x}$ analogously.

(iii) For each $t \in[0,1]$, the function $f(t, \cdot): \mathbb{R}^{M} \rightarrow \mathbb{R}^{M}$ is quasimonotone increasing - that is, if $x_{\mu} \leq y_{\mu}$ for all $\mu \in M$, then

$$
f_{\nu}(t, x) \leq f_{\nu}(t, y) \quad \text { for every } \nu \in M \text { that satisfies } x_{\nu}=y_{\nu} .
$$

(iv) The function $f$ is integrably bounded, in this sense: for each $\mu \in M$ there exists an integrable function $\beta_{\mu}:[0,1] \rightarrow \mathbb{R}$ satisfying $\left|f_{\mu}(t, x)\right| \leq \beta_{\mu}(t)$ for all $t, x$.

Then (IVP) has at least one solution. Moreover, it has a maximal solution - i.e., a solution that is equal to the pointwise maximum of all solutions.

The proof of Theorem 1.1 uses subsolutions, which are interesting in their own right. By a subsolution of (IVP), we shall mean a continuous function $y:[0,1] \rightarrow$ $\mathbb{R}^{M}$ satisfying these conditions for each $\mu$ :

$$
y_{\mu}(0)=0
$$

$y_{\mu}$ is absolutely continuous, and $\left|y_{\mu}^{\prime}(t)\right| \leq \beta_{\mu}(t)$ almost everywhere,

$$
y_{\mu}^{\prime}(s) \leq f_{\mu}(s, y(s)) \text { almost everywhere. }
$$

Clearly, any solution is also a subsolution. Theorem 1.1 follows easily from Theorem 1.2 , below; the proof of Theorem 1.2 will be given in $\S 4$ of this paper.

Theorem 1.2. Under the assumptions of Theorem 1.1, a subsolution of (IVP) exists; the pointwise supremum of any collection of subsolutions is a subsolution; the pointwise supremum of all subsolutions is actually a pointwise maximum, and a solution of (IVP).

\section{Discussion of THE HYPOTHESES}

We have taken the time-interval to be $[0,1]$ and the initial value $x(0)$ to be 0 only for simplicity of notation; our argument would work just as well for any other compact interval and any other initial value. Our results for initial value problems can be converted to results for final value problems by reversing inequalities and multiplying functions by -1 in appropriate places.

Likewise, we have used $[0,1] \times \mathbb{R}^{M}$ as domain mainly for simplicity; a smaller domain can be used with more complicated arguments. For instance, let $c_{\nu}=$ 
$\int_{0}^{1} \beta_{\nu}(s) d s$ and $Q=\prod_{\nu \in M}\left[-c_{\nu}, c_{\nu}\right]$; we could use $[0,1] \times Q$ as a domain. Indeed, suppose $f$ satisfies our four hypotheses on that smaller domain. Let $p_{\nu}: \mathbb{R} \rightarrow$ $\left[-c_{\nu}, c_{\nu}\right]$ be the closest-point projection, and let $P: \mathbb{R}^{M} \rightarrow Q$ be defined coordinatewise by $(P x)_{\nu}=p_{\nu}\left(x_{\nu}\right)$. Define $\tilde{f}(t, x)=f(t, P x)$. Then $\tilde{f}$ satisfies all of hypotheses (i), (ii), (iii), (iv) on $[0,1] \times \mathbb{R}^{M}$. Applying our theorem to $\tilde{f}$ yields the desired conclusion for $f$.

2.1. Measurability. Throughout this paper's theorems and proofs, we work with functions defined everywhere, not just with equivalence classes of functions defined almost everywhere. Thus, hypotheses (ii), (iii), (iv) are stated in terms of all $t$ rather than almost all $t$, and $\beta_{\mu}$ is an "integrable function" rather than a member of $L^{1}[0,1]$. When we say that a condition such as $x_{\mu}^{\prime}(t)=f(t, x(t))$ holds almost everywhere in $[0,1]$, we mean that it holds outside some set $N_{\mu}$ of measure 0 ; different $\mu$ 's may yield different $N_{\mu}$ 's. We do not assert that the set $\bigcup_{\mu \in M} N_{\mu}$ has measure 0 , or even that the set is Lebesgue-measurable; a need for measurability of such sets will not arise in our considerations. By working with only one component $x_{\mu}$ at a time, we shall avoid the complications commonly associated with measure theory in nonseparable spaces.

The terms "superposition-measurable," "supermeasurable," and "superpositionally measurable" can be traced back at least as far as [14 and 26]. More recent papers such as [1], [2], 13] use the abbreviation "sup-measurable," which unfortunately suggests suprema. We are reluctant to alter a terminology that has already been widely used, but perhaps "composition-measurable" would be more descriptive.

As defined in [14, [26], "superposition-measurability" refers to functions $f$ with the property that

$(*)$ whenever $x$ is a measurable function, then $t \mapsto f(t, x(t))$ is measurable.

Our hypothesis (i), which is more immediately relevant to differential equations, is actually equivalent to $(*)$ in finite dimensions. That is proved in [13, [14 for one dimension, but the proof extends without difficulty to finite dimensions. We have taken the liberty of extending the terminology to arbitrary dimensions.

The paper [13] contains a few observations about differential equations, but [1], 2], [13] are primarily concerned with set-theoretic properties of superpositionmeasurability (e.g., with proving that certain unmeasurable sets exist). Some researchers in differential equations who have not followed recent developments in measure theory may be surprised to learn that condition $(*)$ neither implies, nor is implied by, the measurability of $f$. But that is not so surprising when we recall that the "measurability" of $f$ just means that the inverse image of any Borel subset of $\mathbb{R}$ is a Lebesgue-measurable subset of $\mathbb{R}^{2}$. A slightly stronger assumption - that the inverse image of any Borel subset of $\mathbb{R}$ is a Borel subset of $\mathbb{R}^{2}$ - does imply condition $(*)$.

2.2. Quasisemicontinuity. Our hypothesis (ii) was used in [4] with $M=\{1\}$, but in that paper the condition was called "quasi-increasing." Fortunately, that term has not yet appeared in many papers, and can be easily replaced by a more descriptive term. We have now renamed hypothesis (ii) as "quasisemicontinuous," because of its resemblance to conditions of upper- or lower-semicontinuity. 
What happens if we omit the hypothesis of quasisemicontinuity? Partial answers are provided by these two examples, with $M=\{1\}$ :

$$
f(t, x)=\left\{\begin{array}{cl}
1 & (x<0), \\
0 & (x=0), \\
-1 & (x>0),
\end{array} \quad g(t, x)=\left\{\begin{array}{cc}
1 & (x<0), \\
-1 & (x=0), \\
-1 & (x>0)
\end{array}\right.\right.
$$

Both of these functions violate hypothesis (ii), and satisfy all other hypotheses of this paper. With initial condition $x(0)=0$, the differential equation $x^{\prime}(t)=$ $f(t, x(t))$ has a solution, and the differential equation $x^{\prime}(t)=g(t, x(t))$ does not. Thus, hypothesis (ii) is neither completely necessary (in the sense of being implied by the existence of solutions) nor completely superfluous. Perhaps later research will enable us to replace (ii) with a weaker hypothesis.

2.3. Quasimonotonicity. The term "quasimonotonicity" was coined by Walter [30] and can be thought of as a weakened monotonicity condition. According to Walter, this property was first used by Müller [16], and has been found to be a key concept in extending results about differential equations and inequalities from $\mathbb{R}^{1}$ to higher dimensions (see also [12], 27], 28, 32]). A "mixed" quasimonotonicity is used in [5. The paper 20] shows that in some contexts quasimonotonicity is equivalent to a flow-invariance condition. (Further discussion of flow-invariance can be found in $\S 6$ of 22 .) Some other results on quasimonotonicity and more references can be found in [11], 15], [24].

In some contexts it has been shown that quasimonotonicity of $f$ is not only sufficient but also necessary for a Comparison Principle:

If $v(a) \ll w(a)$ and $v^{\prime}(t)-f(t, v(t)) \ll w^{\prime}(t)-f(t, w(t))$ for all $t \in[a, b]$, then $v(b) \ll w(b)$.

Here the relation $x \ll y$ means that $y-x$ lies in that interior of the ordering cone. See [31] for an introduction to this subject in finite dimensions. In infinite dimensions, quasimonotonicity implies the Comparison Principle (see [29]), and a converse holds if $f$ is continuous (see 25]). However, all these results assume the Banach space is ordered by a cone with nonempty interior.

Our own Theorem 1.2 is also a comparison theorem, but of a rather different sort. The ordering cone has empty interior, in $\mathbb{R}^{M}$ when $M$ is infinite, and in most of the alternate spaces considered in $\S 5$. We do not yet know whether our Theorem 1.2 can be reformulated in a fashion that yields a converse.

We do know that quasimonotonicity is not implied by the existence of solutions. That is evident from the following example. Let $M=\{1,2\}$, and let

$$
f\left(t,\left(\begin{array}{l}
x_{1} \\
x_{2}
\end{array}\right)\right)=\left(\begin{array}{c}
-x_{2} \\
0
\end{array}\right) .
$$

Then $f$ is not quasimonotone increasing, but by Peano's Theorem (discussed in $\S 3$ ) we know that (IVP) has a solution. At present we do not know whether hypothesis (iii) is superfluous - i.e., whether existence of solutions is implied by hypotheses (i), (ii), (iv).

2.4. Boundedness. The boundedness hypothesis (iv) is included for simplicity. It can be replaced by weaker but more complicated hypotheses using a variety of standard techniques, establishing local existence instead of global existence - i.e., establishing existence on $[0, T)$ for some $T>0$, instead of on $[0,1]$. Of course, if all boundedness hypotheses are omitted, then we cannot prove global existence, even 
if we strengthen our other hypotheses. For instance, in one dimension, the function $f(t, x)=(x+1)^{3}$ is continuous and increasing, but the unique solution of (IVP) is $x(t)=-1+(1-2 t)^{-1 / 2}$, which blows up as $t$ increases to $\frac{1}{2}$.

\section{Further COMPARISON With THE LITERATURE}

A classical theorem of Peano [17] states that, in finite dimensions, if $f$ is continuous and bounded, then the initial value problem (IVP) has a solution. Carathéodory [6] weakened the joint continuity assumption to measurability plus the assumption that $f(t, x)$ is continuous in its second argument. These results can be found in more recent textbooks on differential equations (e.g., 7]). Finite-dimensionality can be replaced with compactness (e.g., see $\S 30.12$ in [23]). Continuity can be replaced by monotonicity; this was shown by Biles [3] in finite dimensions and extended to infinite dimensions by Schechter in $\S 30.14$ of $[23]$.

A number of papers - e.g., [3], 44, [5], [11], 21], [24 — have established existence of a solution when $f$ satisfies a weakened continuity hypothesis and/or a weakened monotonicity hypothesis. The present paper, continuing to weaken those conditions, extends and subsumes several of those earlier papers. In particular:

- The result of [4] is just the present paper's result specialized to the case of $\operatorname{card}(M)=1$. That is not immediately apparent, since [4] does not explicitly mention any condition like our quasimonotonicity hypothesis (iii). However, with $\operatorname{card}(M)=1$, every function $f:[0,1] \times \mathbb{R} \rightarrow \mathbb{R}$ satisfies our quasimonotonicity hypothesis (iii).

- This paper also generalizes the finite-dimensional result on increasing functions presented in [3], since any increasing function must satisfy our hypotheses (ii) and (iii).

- This paper goes beyond those earlier results. For instance, let $H: \mathbb{R} \rightarrow\{0,1\}$ be the Heaviside function - that is, the characteristic function of the interval $[0,+\infty)$. Let $M=\{1,2\}$, and let

$$
f\left(t,\left(\begin{array}{c}
x_{1} \\
x_{2}
\end{array}\right)\right)=\left(\begin{array}{c}
H\left(x_{2}\right)-\arctan \left(x_{1}\right) \\
H\left(x_{1}\right)-\arctan \left(x_{2}\right)
\end{array}\right) .
$$

It is easy to verify that $f$ is not continuous or increasing, so $f$ does not satisfy the hypotheses of earlier papers. Nevertheless, $f$ satisfies all the hypotheses of the present paper.

Some questions remain open. In particular, we would like to see sufficient conditions for existence of solutions that simultaneously generalize the hypotheses of this paper and the hypotheses of Peano's classical result. (That this paper's result does not contain Peano's result is evident from the example in §2.3.)

There are several substantially different methods for proving existence of solutions to differential equations. This paper's method - taking the supremum of subsolutions - does not involve $\varepsilon$-approximate solutions, and so it is, in some sense, nonconstructive. The technique of subsolutions can be traced back to [3], [10], [30]. As far as we are aware, Peano [17] was the first to use the suprema of subsolutions, in 1886. Another early user of this technique was Perron [18], in 1914. Perron later used it to solve the Dirichlet Problem for $\Delta u=0$; this is at the base of the modern "viscosity solutions" approach to nonlinear partial differential equations.

Our proof also draws heavily on measure-theoretic techniques that were used in one dimension in [21]. We suspect that the proof given here might be reformulated 
in terms of a fixed-point argument such as that in [19] or that in $\S 30.14$ of [23], but so far we have not gained any insights from those approaches.

\section{Proof of the MAIN THEOREM}

Let $B(t)=\int_{0}^{t} \beta(s) d s$ - that is, let $B_{\mu}(t)=\int_{0}^{t} \beta_{\mu}(s) d s$ for each $\mu$. Then each $B_{\mu}$ is absolutely continuous and increasing. Whenever we mention an interval $[s, t]$, it will be understood that $s \leq t$; hence $B_{\mu}(s) \leq B_{\mu}(t)$.

We first note that $(2)$ is equivalent to

$$
\left|y_{\mu}(t)-y_{\mu}(s)\right| \leq B_{\mu}(t)-B_{\mu}(s) \text { for all }[s, t] \subseteq[0,1] .
$$

Also, for a function $y$ whose components are absolutely continuous, condition (3) is equivalent to

$$
y_{\mu}(t)-y_{\mu}(s) \leq \int_{s}^{t} f_{\mu}(r, y(r)) d r \quad \text { for all }[s, t] \subseteq[0,1]
$$

Let $\Xi$ be the set of all subsolutions of (IVP). It is easy to see that

The set $\Xi$ of all subsolutions is nonempty. In fact, $-B \in \Xi$.

The rest of our argument is given in two propositions.

Proposition 4.1. The pointwise supremum of any nonempty subset of $\Xi$ is also a member of $\Xi$. (In particular, $\Xi$ has a maximum member - namely, the supremum of $\Xi$.)

Proof. Let $S$ be any nonempty subset of $\Xi$. Then $S$ is bounded componentwise, by hypothesis (iv). Let $\sigma$ be the pointwise supremum of $S$ - i.e., define $\sigma:[0,1] \rightarrow \mathbb{R}^{M}$ by taking $\sigma_{\mu}(t)=\sup \left\{y_{\mu}(t): y \in S\right\}$ for each $t \in[0,1]$ and each $\mu \in M$. We are to show that $\sigma \in \Xi$.

Obviously $\sigma$ satisfies (1).

To prove that $\sigma$ satisfies $\left(2^{\prime}\right)$, fix any $y \in S$ and any $s, t \in[0,1]$ (not necessarily assuming $s<t$ or $t<s$ ). Then

$$
y_{\mu}(s) \leq\left|y_{\mu}(s)-y_{\mu}(t)\right|+y_{\mu}(t) \leq\left|B_{\mu}(s)-B_{\mu}(t)\right|+\sigma_{\mu}(t) .
$$

Now take the supremum on the left side; this yields $\sigma_{\mu}(s) \leq\left|B_{\mu}(s)-B_{\mu}(t)\right|+\sigma_{\mu}(t)$. A similar argument yields $\sigma_{\mu}(t) \leq\left|B_{\mu}(t)-B_{\mu}(s)\right|+\sigma_{\mu}(s)$. Combine those results to obtain $\left|\sigma_{\mu}(s)-\sigma_{\mu}(t)\right| \leq\left|B_{\mu}(s)-B_{\mu}(t)\right|$. Thus each $\sigma_{\mu}$ is absolutely continuous, and $\sigma$ satisfies $\left(2^{\prime}\right)$.

It remains to show that $\sigma$ satisfies (3). We first prove this under the additional assumption that $S$ has just two members; say $S=\{v, w\}$. Fix any $\mu$; then $\sigma_{\mu}(t)=$ $\max \left\{v_{\mu}(t), w_{\mu}(t)\right\}$, and we are to show that $\sigma_{\mu}^{\prime}(t) \leq f_{\mu}(t, \sigma(t))$ almost everywhere. We already know that $\sigma_{\mu}, v_{\mu}, w_{\mu}$ are differentiable almost everywhere; and since $v, w \in \Xi$, we know

$$
v_{\mu}^{\prime}(t) \leq f_{\mu}(t, v(t)) \quad \text { and } \quad w_{\mu}^{\prime}(t) \leq f_{\mu}(t, w(t))
$$

for almost all $t$. Fix any $t$ where both of those inequalities hold, and where all three functions $\sigma_{\mu}, v_{\mu}, w_{\mu}$ are differentiable; it suffices to show that $\sigma_{\mu}^{\prime}(t) \leq$ $f_{\mu}(t, \sigma(t))$ at that particular $t$. By the quasimonotonicity of $f$, we know that $\max \left\{f_{\mu}(t, v(t)), f_{\mu}(t, w(t))\right\} \leq f_{\mu}(t, \sigma(t))$.

If $v_{\mu}(t)<w_{\mu}(t)$, then $\sigma_{\mu}=w_{\mu}$ on some neighborhood of $t$; hence $\sigma_{\mu}^{\prime}(t)=$ $w_{\mu}^{\prime}(t) \leq f_{\mu}(t, w(t)) \leq f_{\mu}(t, \sigma(t))$. A similar argument applies if $v_{\mu}(t)>w_{\mu}(t)$. Thus we may assume $v_{\mu}(t)=w_{\mu}(t)=\sigma_{\mu}(t)$. 
If $v_{\mu}^{\prime}(t)<w_{\mu}^{\prime}(t)$, then $\sigma_{\mu}$ agrees with $v_{\mu}$ on an interval to the left of $t$ and with $w_{\mu}$ to the right of $t$; but then $\sigma_{\mu}^{\prime}(t)$ does not exist. Similarly if $v_{\mu}^{\prime}(t)>w_{\mu}^{\prime}(t)$. Thus $v_{\mu}^{\prime}(t)$ and $w_{\mu}^{\prime}(t)$ are equal. It follows easily that their common value is also equal to $\sigma_{\mu}^{\prime}(t)$. Then $\sigma_{\mu}^{\prime}(t)=w_{\mu}^{\prime}(t) \leq f_{\mu}(t, w(t)) \leq f_{\mu}(t, \sigma(t))$.

Thus, we have shown that the supremum of two members of $\Xi$ belongs to $\Xi$. By induction, the supremum of finitely many members of $\Xi$ belongs to $\Xi$. Now let $S$ be any infinite subset of $\Xi$; it suffices to show that $\sigma$ satisfies $\left(3^{\prime}\right)$.

Fix any $\mu \in M$. Let $r_{1}, r_{2}, r_{3}, \ldots$ be an enumeration of the rationals in $[0,1]$. Since $\sigma$ is the supremum of $S$, for each pair of positive integers $j$ and $k$ we can find some $z=z^{j, k} \in S$ satisfying $z_{\mu}\left(r_{j}\right) \leq \sigma_{\mu}\left(r_{j}\right) \leq z_{\mu}\left(r_{j}\right)+\frac{1}{k}$. Let $y^{n}$ be the supremum of the finite set $\left\{z^{j, k}: j, k \leq n\right\}$; then $y^{n} \in \Xi$. Thus $y^{1}, y^{2}, y^{3}, \ldots$ is an increasing sequence in $\Xi$, so $y^{\infty}=\lim _{n \rightarrow \infty} y^{n}=\sup _{n} y^{n}=\sup _{j, k} z^{j, k}$ exists coordinatewise. By results that we have already established, the function $y^{\infty}$ satisfies $\left(2^{\prime}\right)$, so $y^{\infty}$ is continuous and the function $f\left(s, y^{\infty}(s)\right)$ is measurable. By our construction involving $r_{j}$ 's, we have $y_{\mu}^{\infty}(r)=\sigma_{\mu}(r)$ for every rational number $r \in[0,1]$. Since $y^{\infty}$ and $\sigma$ are both continuous, we have $y_{\mu}^{\infty}=\sigma_{\mu}$. We also know that $y_{\nu}^{\infty} \leq \sigma_{\nu}$ for every $\nu \in M$, since $\sigma=\sup S$ and $y^{\infty}$ is the supremum of a subset of $S$.

Now fix any subinterval $[s, t] \subseteq[0,1]$, and compute

$$
\begin{aligned}
\sigma_{\mu}(t) & -\sigma_{\mu}(s)=\lim _{n \rightarrow \infty}\left[y_{\mu}^{n}(t)-y_{\mu}^{n}(s)\right] \leq \limsup _{n \rightarrow \infty} \int_{s}^{t} f_{\mu}\left(r, y^{n}(r)\right) d r \\
& \leq \int_{s}^{t} \limsup _{n \rightarrow \infty} f_{\mu}\left(r, y^{n}(r)\right) d r \leq \int_{s}^{t} f_{\mu}\left(r, y^{\infty}(r)\right) d r \leq \int_{s}^{t} f_{\mu}(r, \sigma(r)) d r .
\end{aligned}
$$

Here the four inequalities follow from (respectively) $y^{n}$ being a member of $\Xi$, Fatou's Lemma, the quasisemicontinuity of $f$, and the quasimonotonicity of $f$. This shows that $\sigma$ satisfies $\left(3^{\prime}\right)$ and completes the proof of Proposition 4.1.

Proposition 4.2. Let $\sigma=\max \Xi$. Then $\sigma$ is a solution of (IVP).

Proof. By Proposition 4.1, we know $\sigma$ is a subsolution of (IVP). Suppose $\sigma$ is not a solution. Then there exist some $\mu \in M$ and some measurable set $E \subseteq(0,1)$ with positive measure, such that $\sigma_{\mu}^{\prime}(t)<f_{\mu}(t, \sigma(t))$ for all $t \in E$. For each $k \in \mathbb{N}$ let

$$
E_{k}=\left\{t \in E: f_{\mu}(t, \sigma(t))>\sigma_{\mu}^{\prime}(t)+\frac{4}{k}\right\} .
$$

Then each $E_{k}$ is measurable, and $E=\bigcup_{k} E_{k}$; hence there is some $k_{0} \in \mathbb{N}$ such that $E_{k_{0}}$ has positive measure.

Let $e \in \mathbb{R}^{M}$ be the characteristic function of the singleton $\{\mu\}$ - i.e., let $e_{\mu}=1$ and $e_{\nu}=0$ for all $\nu \in M \backslash\{\mu\}$. Let $s$ be a positive number. We claim that the real-valued function

$$
g(t)=\inf _{0 \leq r \leq s} f_{\mu}(t, \sigma(t)+r e)
$$

is measurable. To see this, temporarily fix any number $p$; it suffices to show that the set $S=\{t \in[0,1]: g(t) \geq p\}$ is measurable. Any number $r \in[0, s]$ can be approximated from below by rational numbers $r \in[0, s]$; hence by the limsup property in hypothesis (ii), the set $S$ is also equal to

$$
\bigcap_{r \in[0, s] \cap \mathbb{Q}}\left\{t \in[0,1]: f_{\mu}(t, \sigma(t)+r e) \geq p\right\} .
$$


That set is the intersection of countably many measurable sets, so it is measurable. This proves the claim. Therefore each of the sets

$$
F_{m}=\left\{t \in(0,1): \inf _{0 \leq r \leq 1 / m} f_{\mu}(t, \sigma(t)+r e)>f_{\mu}(t, \sigma(t))-\frac{1}{k_{0}}\right\}
$$

(for $m=1,2,3, \ldots$ ) is measurable.

By hypothesis (ii), we have $\liminf _{r \downarrow 0} f_{\mu}\left(t, z_{0}+r e\right) \geq f_{\mu}\left(t, z_{0}\right)$ for any fixed $t \in(0,1)$ and $z_{0} \in \mathbb{R}^{M}$. Hence for any integer $m$ sufficiently large,

$$
\inf _{0 \leq r \leq 1 / m} f_{\mu}\left(t, z_{0}+r e\right)>f_{\mu}\left(t, z_{0}\right)-\frac{1}{k_{0}}
$$

It follows that $(0,1)=\bigcup_{m=1}^{\infty} F_{m}$.

Then $E_{k_{0}} \cap F_{m_{0}}$ has positive measure for some positive integer $m_{0}$. Let $G=$ $E_{k_{0}} \cap F_{m_{0}}$. We have now shown that there exist some numbers $k_{0}, m_{0} \in \mathbb{N}$ and a measurable set $G \subseteq(0,1)$ with positive measure such that whenever $t \in G$, then

$$
\frac{1}{k_{0}}+\inf _{0 \leq r \leq 1 / m_{0}} f_{\mu}(t, \sigma(t)+r e)>f_{\mu}(t, \sigma(t))>\sigma_{\mu}^{\prime}(t)+\frac{4}{k_{0}} .
$$

Let $\complement G=[0,1] \backslash G$, and let $1_{G}$ and $1_{\complement G}$ denote the characteristic functions of $G$ and $\complement G$ respectively. Let $\lambda$ denote Lebesgue measure. Lebesgue's theorem on differentiation says that if $h$ is any integrable function, then $\lim _{t \rightarrow t_{0}} \frac{1}{t-t_{0}} \int_{t_{0}}^{t} h(s) d s=h\left(t_{0}\right)$ for almost every $t_{0}$. In particular,

$$
\lim _{t \downarrow t_{0}} \frac{\int_{t_{0}}^{t} 1_{G}(s) d s}{t-t_{0}}=1_{G}\left(t_{0}\right) \quad \text { and } \quad \lim _{t \downarrow t_{0}} \frac{\int_{t_{0}}^{t} 1_{\complement G}(s) \beta_{\mu}(s) d s}{t-t_{0}}=1_{\complement G}\left(t_{0}\right) \beta_{\mu}\left(t_{0}\right)
$$

for almost every $t_{0}$. Since $G$ has positive measure, there is some point $t_{0} \in G$ satisfying those equations. At that point we have $1_{G}\left(t_{0}\right)=1$ and $1_{C G}\left(t_{0}\right)=0$, so

$$
\lim _{t \downarrow t_{0}} \frac{\lambda\left(G \cap\left[t_{0}, t\right]\right)}{t-t_{0}}=1 \quad \text { and } \quad \lim _{t \downarrow t_{0}} \frac{\int_{\left[t_{0}, t\right] \backslash G} \beta_{\mu}(s) d s}{t-t_{0}}=0 .
$$

Also, since $\beta_{\mu}$ is integrable, $\lim _{t \downarrow t_{0}} \int_{t_{0}}^{t} \beta_{\mu}(s) d s=0$. From those three limit statements, it is clear that there is some $\delta>0$ such that every $t \in\left(t_{0}, t_{0}+\delta\right)$ will satisfy

$$
\frac{\lambda\left(G \cap\left[t_{0}, t\right]\right)}{t-t_{0}}>\frac{1}{2}, \quad \frac{\int_{\left[t_{0}, t\right] \backslash G} \beta_{\mu}(s) d s}{t-t_{0}}<\frac{1}{4 k_{0}}, \quad \int_{t_{0}}^{t} \beta_{\mu}(s) d s<\frac{1}{2 m_{0}} .
$$

Now define $w:[0,1] \rightarrow \mathbb{R}^{M}$ by taking $w_{\nu}=\sigma_{\nu}$ for all $\nu \neq \mu$, and taking $w_{\mu}$ absolutely continuous with $w_{\mu}(0)=0$ and

$$
w_{\mu}^{\prime}(t)=\left\{\begin{array}{cll}
\sigma_{\mu}^{\prime}(t) & \text { for } \quad t \in\left(0, t_{0}\right), \\
\sigma_{\mu}^{\prime}(t)+\frac{3}{k_{0}} & \text { for } \quad t \in\left(t_{0}, t_{0}+\delta\right) \cap G, \\
-\beta_{\mu}(t) & \text { for } t \in\left(t_{0}, t_{0}+\delta\right) \backslash G, \\
-\beta_{\mu}(t) & \text { for } t \in\left(t_{0}+\delta, t_{1}\right), \\
\sigma_{\mu}^{\prime}(t) & \text { for } t \in\left(t_{1}, 1\right),
\end{array}\right.
$$


where $t_{1}$ will be specified below. Note that $w_{\mu}=\sigma_{\mu}$ on $\left[0, t_{0}\right]$. For $t \in\left(t_{0}, t_{0}+\delta\right)$, we can now compute

$$
\begin{aligned}
w_{\mu}(t) & =\sigma_{\mu}\left(t_{0}\right)+\int_{t_{0}}^{t} w_{\mu}^{\prime}(s) d s \\
& =\sigma_{\mu}\left(t_{0}\right)+\int_{\left[t_{0}, t\right] \cap G}\left[\sigma_{\mu}^{\prime}(s)+\frac{3}{k_{0}}\right] d s-\int_{\left[t_{0}, t\right] \backslash G} \beta_{\mu}(s) d s \\
& =\sigma_{\mu}(t)-\int_{\left[t_{0}, t\right] \backslash G} \sigma_{\mu}^{\prime}(s) d s-\int_{\left[t_{0}, t\right] \backslash G} \beta_{\mu}(s) d s+\frac{3}{k_{0}} \lambda\left(\left[t_{0}, t\right] \cap G\right) \\
& \geq \sigma_{\mu}(t)-2 \int_{\left[t_{0}, t\right] \backslash G} \beta_{\mu}(s) d s+\frac{3}{k_{0}} \lambda\left(\left[t_{0}, t\right] \cap G\right) \quad \text { using }(2) \\
\geq & \sigma_{\mu}(t)+\frac{1}{k_{0}}\left(t-t_{0}\right) \quad \text { using }(5) .
\end{aligned}
$$

Thus

$$
w_{\mu}(t)>\sigma_{\mu}(t) \quad \text { for all } t \in\left(t_{0}, t_{0}+\delta\right) .
$$

However, we shall show that $w \in \Xi$, contradicting our construction of $\sigma$ as the supremum of $\Xi$.

Let $\psi(t)=w_{\mu}\left(t_{0}+\delta\right)-\sigma_{\mu}\left(t_{0}+\delta\right)+\int_{t_{0}+\delta}^{t}\left[-\beta_{\mu}(s)-\sigma_{\mu}^{\prime}(s)\right] d s$. Then $\psi\left(t_{0}+\delta\right)>0$, and $\psi$ is decreasing on $\left(t_{0}+\delta, 1\right]$. Define $t_{1}$ to be the lowest number in $\left(t_{0}+\delta, 1\right)$ that satisfies $\psi\left(t_{1}\right)=0$, or let $t_{1}=1$ if $\psi>0$ everywhere in $\left(t_{0}+\delta, 1\right)$. It follows that

$$
w_{\mu}>\sigma_{\mu} \text { on }\left(t_{0}, t_{1}\right), \quad w_{\mu}=\sigma_{\mu} \text { elsewhere in }(0,1) .
$$

To show that $w \in \Xi$, we begin by observing that $w$ satisfies (1).

To verify (2), we must show that $\left|w_{\nu}^{\prime}(t)\right| \leq \beta_{\nu}(t)$ for all $\nu$ and almost all $t$. This is obvious for $\nu \neq \mu$, and for $\nu=\mu$ when $t$ is outside the set $G \cap\left(t_{0}, t_{0}+\delta\right)$. For $\nu=\mu$ with $t \in G \cap\left(t_{0}, t_{0}+\delta\right)$, we have

$$
\beta_{\mu}(t)>\beta_{\mu}(t)-\frac{1}{k_{0}} \geq f_{\mu}(t, \sigma(t))-\frac{1}{k_{0}}>\sigma_{\mu}^{\prime}(t)+\frac{3}{k_{0}} \geq-\beta_{\mu}(t)+\frac{3}{k_{0}}>-\beta_{\mu}(t) .
$$

Since $w_{\mu}^{\prime}(t)=\sigma_{\mu}^{\prime}(t)+\frac{3}{k_{0}}$ on $G \cap\left(t_{0}, t_{0}+\delta\right)$, we have $\beta_{\mu}(t) \geq w_{\mu}^{\prime}(t) \geq-\beta_{\mu}(t)$. This proves (2).

It remains only to verify $(3)$ - that is, to show that $w_{\nu}^{\prime}(s) \leq f_{\nu}(s, w(s))$ almost everywhere for each $\nu$. We establish this in several cases:

In the cases of $\nu \neq \mu$ we may compute

$$
w_{\nu}^{\prime}(t)=\sigma_{\nu}^{\prime}(t) \leq f_{\nu}(t, \sigma(t)) \leq f_{\nu}(t, w(t))
$$

where the last inequality follows from (6) and quasimonotonicity. Thus, it remains to prove $w_{\mu}^{\prime}(s) \leq f_{\mu}(s, w(s))$.

That inequality clearly holds on the intervals $\left(0, t_{0}\right)$ and $\left(t_{1}, 1\right)$, since on those intervals $w=\sigma$. It also holds on the sets $\left(t_{0}, t_{0}+\delta\right) \backslash G$ and $\left(t_{0}+\delta, t_{1}\right)$, since on those sets $w_{\mu}^{\prime}(s)=-\beta_{\mu}(s) \leq f_{\mu}(s, w(s))$.

Finally, on the set $G \cap\left(t_{0}, t_{0}+\delta\right)$, we may reason as follows: We have

$$
w_{\mu}(t)-\sigma_{\mu}(t)=\int_{t_{0}}^{t}\left[w_{\mu}^{\prime}(s)-\sigma_{\mu}^{\prime}(s)\right] d s \leq 2 \int_{t_{0}}^{t} \beta_{\mu}(s) d s \leq \frac{1}{m_{0}}
$$


using inequality (5). From this result and (6) we see that $w(t)=\sigma(t)+r e$ for some $r \in\left[0, \frac{1}{m_{0}}\right]$. Therefore, using both parts of (4),

$$
w_{\mu}^{\prime}(t)=\sigma_{\mu}^{\prime}(t)+\frac{3}{k_{0}}<f_{\mu}(t, \sigma(t))-\frac{1}{k_{0}} \leq f_{\mu}(t, w(t)) .
$$

This completes the proof.

\section{Remarks about Further Generalizations}

For simplicity we have presented our main arguments only in the setting of $\mathbb{R}^{M}$. However, the vector lattice $\mathbb{R}^{M}$ used in the main theorem of this paper can be replaced by certain Dedekind-complete Banach lattices such as $c_{0}$ or $\ell_{p}$ $(1 \leq p \leq \infty)$, with only trivial changes in the statement and proof of the theorem. When these lattices are used, we can use the norm topology instead of the product topology, and Bochner integrals instead of componentwise Lebesgue integrals. We emphasize that, with the exception of $\ell_{\infty}$, these lattices are ordered by cones with empty interior.

Some limitations in our methods prevent us from considering vector lattices in general. Our proof of Proposition 4.1 uses the coordinatewise representation of vectors in $\mathbb{R}^{M}$, not just for convenience of notation, but also for the fact that the coordinatewise functionals $\varphi(x)=x_{\mu}$ satisfy $\varphi(v \vee w)=\max \{\varphi(v), \varphi(w)\}$. An analogous property is not valid for some vector lattices - e.g., the lattice of all real-valued measures on a $\sigma$-algebra. See $\S 11.47-11.48$ and $\S 21.11 . c$ of [23].

Dedekind completeness of the Banach lattice $(X,\|\|, \preceq)$ is needed to assure that $\sigma(t)=\sup \{y(t): y \in S\}$ exists in the proof of Proposition 4.1. (Thus our methods would not apply to $X=C[0,1]$.) For the same reason, hypothesis (iv) must be replaced by the condition

$\left(\right.$ iv' $\left.^{\prime}\right)$ There exist Bochner-integrable functions $\alpha, \beta:[0,1] \rightarrow X$ such that $\alpha(t) \preceq$ $f(t, x) \preceq \beta(t)$ for all $t, x$.

(A similar hypothesis was used in $\S 30.14$ of [23].) One might be tempted to try to use the slightly weaker hypothesis

$\left(\right.$ iv $\left.^{\prime \prime}\right)$ There exists an integrable function $\beta:[0,1] \rightarrow \mathbb{R}$ such that $\|f(t, x)\| \leq \beta(t)$ for all $t, x$.

However, that assumption is not strong enough to guarantee existence of solutions, even if we strengthen our other hypotheses. That is evident from the following modification of the well-known example of Dieudonné 8]. Let $X=c_{0}=$ \{sequences of reals convergent to 0$\}$, with its usual sup norm. Let

$$
\varphi(x)=\left\{\begin{array}{ll}
0 & (x \leq 0), \\
x & (0 \leq x \leq 1), \\
1 & (1 \leq x),
\end{array} \quad f, \quad\left[\begin{array}{c}
x_{1} \\
x_{2} \\
x_{3} \\
\vdots
\end{array}\right]\right)=\left[\begin{array}{c}
\sqrt[3]{1+\varphi\left(x_{1}\right)} \\
\sqrt[3]{\frac{1}{2}+\varphi\left(x_{2}\right)} \\
\sqrt[3]{\frac{1}{3}+\varphi\left(x_{3}\right)} \\
\vdots
\end{array}\right]
$$

Then $f: \mathbb{R} \times c_{0} \rightarrow c_{0}$ is increasing in its second argument, continuous, and bounded in norm by $\sqrt[3]{2}$. Hence it satisfies all of our hypotheses (i), (ii), (iii) 
mutatis mutandis, as well as hypothesis (iv"). However, (IVP) has no solution in $c_{0}$; that can be shown as follows: If (IVP) had a solution, it would have to be $x(t)=\left(x_{1}(t), x_{2}(t), x_{3}(t), \ldots\right)$, where $x_{j}(t)$ is the solution of the $j$ th component problem. Since $x_{j}(0)=0$ and $\|f(t, x)\| \leq \sqrt[3]{2}$ for all $t, x$, we have $0 \leq x_{j}(t) \leq 1$ at least for all $t$ in $\left[0,2^{-1 / 3}\right]$. On that interval, we have $\varphi\left(x_{j}(t)\right)=x_{j}(t)$ and so $x_{j}^{\prime}(t)=\sqrt[3]{\frac{1}{j}+x_{j}(t)}$, which has unique solution $x_{j}(t)=\left(\frac{2}{3} t+j^{-2 / 3}\right)^{3 / 2}-\frac{1}{j}$. But then the sequence $x(t)$ does not lie in $c_{0}$ when $0<t \leq 2^{-1 / 3}$.

\section{REFERENCES}

1. M. Balcerzak, Some results on superposition-measurability, Real Analysis Exchange 17 (1991/ 1992), 597-607. MR 93k:28001

2. M. Balcerzak and K. Ciesielski, On the sup-measurable functions problem, Real Anal. Exchange 23 (2) (1997-98), 787-797. CMP 98:17

3. Daniel C. Biles, Existence of solutions for discontinuous differential equations, Differential and Integral Equations 8 (1995), 1525-1532. MR 96d:34003

4. Daniel C. Biles and Paul A. Binding, On Carathéodory's conditions for the initial value problem, Proceedings of the American Mathematical Society 125 (1997), 1371-1376. MR 97g:34008

5. L. P. Burton and William M. Whyburn, Minimax solutions of ordinary differential systems, Proceedings of the American Mathematical Society 3 (1952), 794-803. MR 14:470i

6. C. Carathéodory, Vorlesungen über reelle Funktionen, Leipzig, 1927; reprinted, New York, 1948, pp. 665-688. MR 37:1530

7. Earl A. Coddington and Norman Levinson, Theory of Ordinary Differential Equations, McGraw-Hill, New York, 1955. MR 16:1022b

8. J. Dieudonné, Deux examples singuliers d'équations différentielles, Acta Sci. Math. (Szeged) 12B (1950), 38-40. MR 11:729d

9. N. Dunford and J. T. Schwartz, Linear Operators, Part I: General Theory, Pure Appl. Math. 7, Wiley Interscience, New York, 1957. MR 22:8302

10. G. S. Goodman, Subfunctions and the initial-value problem for differential equations satisfying Carathéodory's hypotheses, Journal of Differential Equations 7 (1970), 232-242. MR 41:540

11. Seppo Heikkilä and V. Lakshmikantham, On first order differential equations in ordered Banach spaces, in Inequalities and Applications, ed. by R. P. Agarwal, World Scientific, New Jersey, 1994, p. 293-301. MR 95g:34095

12. E. Kamke, Zur Theorie der Systeme gewöhnlicher Differentialgleichungen II, Acta Mathematica 58 (1932), 57-85.

13. A. B. Kharazishvili, Sup-measurable and weakly sup-measurable mappings in the theory of ordinary differential equations, J. Appl. Anal. 3 (1997), 211-223. MR 99e:28003

14. M. A. Krasnosel'skiı̌, P. P. Zabreı̌ko, et al., Integral operators in spaces of summable functions (Russian), "Nauka," Moscow, 1966. Translated to English by T. Ando, in Monographs and Textbooks on Mechanics of Solids and Fluids, Mechanics: Analysis. Noordhoff International Publishing, Leiden, 1976. MR 52:6505

15. Roland Lemmert, Existenzsätze für gewöhnliche Differentialgleichungen in geordneten Banachräumen, Funkcialaj Ekvacioj 32 (1989), 243-249. MR 90i:34096

16. Max Müller, Über das Fundamentaltheorem in der Theorie der gewöhnlichen Differentialgleichungen, Mathematische Zeitschrift 26 (1926), 619-645.

17. G. Peano, Sull'inegrabilitá delle equazione differenziali di primo ordine, Atti della Accademia delle Scienze di Torino 21 (1885-1886), 677-685.

18. O. Perron, Ein neuer Existenzbeweis für die Integrale der Differentialgleichung $y^{\prime}=f(x, y)$, Math. Annl. 76 (1914), 471-484.

19. Irene Redheffer and Peter Volkmann, Ein Fixpunktsatz für quasimonoton wachsende Funktionen, Arch. der Math. 70 (1998), 307-312. [MR 99a:47086

20. Ray M. Redheffer and Wolfgang Walter, Flow-invariant sets and differential inequalities in normed spaces, Applicable Analysis 5 (1975), 149-161. MR 57:10155 
21. Witold Rzymowski and Dariusz Walachowski, One-dimensional differential equations under weak assumptions, Journal of Mathematical Analysis and Applications 198 (1996), 657-670. MR 97c:34009

22. E. Schechter, A survey of local existence theories for abstract nonlinear initial value problems, pp. 136-184 in Nonlinear Semigroups, Partial Differential Equations, and Attractors (proc. sympos. Washington 1987), ed. by T. L. Gill and W. W. Zachary, Lecture Notes in Math. 1394, Springer-Verlag, Berlin, 1989. MR 91c:34020

23. E. Schechter, Handbook of Analysis and its Foundations, Academic Press, San Diego, 1997, pp. 824-826. MR 98b:00009

24. Alice Chaljub-Simon, Roland Lemmert, Sabina Schmidt, and Peter Volkmann, Gewöhnliche Differentialgleichungen mit quasimonoton wachsenden rechten Seiten in geordneten Banachräumen, pp. 307-320 in General Inequalities 6, ed. by Wolfgang Walter, International Series of Numerical Mathematics 103, Birkhäuser Verlag, Basel, 1992. MR 94c:34092

25. Alice Simon and Peter Volkmann, Remark on quasimonotonicity, in Inequalities and Applications, ed. by R. P. Agarwal, World Scientific, New Jersey, 1994, p. 543-548. MR 95h:34024

26. J. W. Šragin, Conditions for measurability of superpositions (Russian), Dokl. Akad. Nauk SSSR 197 (1971), 295-298; translated in Soviet Math. Dokl. 12 (1971), 465-470.

27. J. Szarski, Sur un système d'inéqalités différentielles, Ann. Soc. Polon. Math. 20 (1947), 126-134. MR 10:121g

28. J. Szarski, Sur les systèmes majorants d'équations différentielles ordinaires, Ann. Soc. Polon. Math. 23 (1950), 206-223. MR 12:705b

29. Peter Volkmann, Gewöhnliche Differentialungleichungen mit quasimonoton wachsenden Funktionen in topologischen Vektorraümen, Mathematische Zeitschrift 127 (1972), 157-164. MR 46:7661

30. Wolfgang Walter, Differential and Integral Inequalities, Springer-Verlag, New York, 1970. Translation of Differential- und Integral-Ungleichungen, Springer, Berlin, 1964. MR 42:6391

31. W. Walter, Ordinary Differential Equations, Graduate Texts in Mathematics 182, Springer, New York, 1998. CMP 98:15

32. T. Ważewski, Systèmes des équations et des inégalités différentielles ordinaires aux deuxièmes membres monotones et leurs applications, Ann. Soc. Polon. Math. 23 (1950), 112-166. MR 12:705a

Department of Mathematics, Western Kentucky University, Bowling Green, KenTUCKY 42101-3576

E-mail address: Daniel.Biles@wku.edu

Department of Mathematics, Vanderbilt University, Nashville, Tennessee 37240-0001

E-mail address: schectex@math.vanderbilt.edu 